\title{
The many faces of the computer: An analysis of clinical software in the primary care consultation
}

\author{
Christopher Pearce ${ }^{a, b, *}$, Michael Arnold ${ }^{c}$, Christine B. Phillips ${ }^{d}$, \\ Stephen Trumble ${ }^{e}$, Kathryn Dwan ${ }^{f}$ \\ a Inner East Melbourne Medicare Local, Australia \\ $\mathrm{b}$ Adjunct Associate Professor, Monash University, Australia \\ c Department of History and Philosophy of Science, University of Melbourne, Australia \\ d Academic Unit of General Practice and Community Health, Australian National University, Australia \\ e Medical Education Unit, University of Melbourne, Australia \\ ${ }^{\mathrm{f}}$ Australian Primary Health Care Research Institute, Australian National University, Australia
}

\section{A R T I C L E I N F O}

\section{Article history:}

Received 8 February 2011

Received in revised form

10 January 2012

Accepted 10 January 2012

Keywords:

Electronic health record

Physician-patient relationship

Medical informatics

Data display

Office visits

\begin{abstract}
A B S T R A C T
Background: Almost all general practitioners in Australia now use a computer for some part of the consultation, and mostly use one of eight clinical software applications. There has been little research into the impact of clinical software on the clinical consultation. Clinical software broadly functions in two ways: it replaces the paper record of the patient's history of health and clinical contacts within the general practice, and it communicates directly to the doctor in various ways about outstanding clinical actions.

Aim: This paper draws on Goffman's notion of "face" to explore the way in which the actions, visual presentation, and interactions between general practitioners, patients and the computer can imbue the software with its own "face" in the consultation.

Methods: Analysis of 141 consultations by 20 doctors (13 men, 7 women), who used one of four medical software applications commonly used in Australian general practice. Consultations were videotaped, tagged, analysed using a hermeneutic framework.

Results: All four software packages replicated constitutive elements of the paper health record, such as medical history, current medications, and the patient's social history, but also introduced other content not present in a paper system. They differed in their use of communicative strategies. This necessitated differing interactions between the software and the doctor. The differences in communicative work of each software package led to their different "faces", along a gradient from a relatively passive mode that provided context dependent information in an unobtrusive way, to a relatively active mode that interrupted to provide information and to demand responses. We conclude that the more active the mode of presence of the computer in the consultation, the more patients and doctors may have to adapt their communicative styles in response.
\end{abstract}

(c) 2012 Elsevier Ireland Ltd. All rights reserved.

* Corresponding author at: Inner East Melbourne Medicare Local, Australia.

E-mail address: drchrispearce@mac.com (C. Pearce). 
1.

\section{Introduction}

Primary care in the developed world is becoming computerised, often in advance of other parts of the health care sector. In Australia, $89 \%$ of general practitioners (GPs) use a computer for some clinical function [1], figures that are mirrored in other jurisdictions [2-4]. Only in the US is this trend reversed, with family physicians lagging behind hospitals [5]. Nevertheless, computerisation of the healthcare space is seen as the way of the future with benefits to be realised in terms of costs, quality and safety [6,7], and for this reason are encouraged by government [8].

The computer is clearly seen to make a significant difference, yet there has been little research into the impact of computers, and none on the impact of different clinical software applications on the clinical consultation. One study examined the design preferences of Romanian primary care physicians [9] for a national system, and another study has tried to develop a coding system for future use [10]. We know that the nature of both the physical setup [11] and the workings of the clinical software influence the consultation [12]. Much work has gone into understanding the human perspectives [13] and into the structure of records [14], but not on the elements of the interaction. Any new technology is often introduced to its users on the basis of its similarities to the technology it supersedes - thus cars were first described as "horseless carriages", television was "radio with pictures", a word-processor was an "electronic typewriter". It is only later that the significant differences, and the implications of those differences, become apparent. Clinical packages are no different, and their design is based on that which they replace: paper records. It is now apparent that they go well beyond the paper record, with the ability to store a wider range of information and the ability to actively interact with the human participants.

Several writers have characterised the consultation in which a computer is used as a three-way interaction $[15,16]$. The exact role of the computer in the consultation has been less clearly theorised. In this paper, we draw on Goffman's notion of "face" to explore the ways in which a software application can constitute a "face" in a consultation.

Most discussion of face draws on Goffman's conceptualisation of face as "an image of self, delineated in terms of approved social attributes" [17], using spoken words and unspoken acts derived in part from assumptions about the roles taken by the various actors in the consultation. Face, for Goffman, was constructed and reconstructed by individuals at the intersection of the social world and the individual. The central concept is that face is not just what is presented, but also must consider the interpretation applied by the receiver. In our context, this means that the computer can exhibit 'face' passively, by creating a situation to which the humans can respond.

Such behaviour has already been noted, in observational studies of the human computer interactions in the consultation. The mere presence of the computer has been described as being a 'magic box' [18], used by the doctor as support for their position. Patients, too, can use the computer's presence to support their position [19]. The computer is changing the nature of what was a dyadic relationship into a triadic one [20].

A consultation between a patient and a doctor is an example of a small drama in which two actors follow scripts, and present a face appropriate to the consultation. To understand this drama, we need to study "the syntactical relations among acts of different persons mutually present to one another" [17]. These syntactical relations have been studied in the medical consultation [21], and these consultations are typically framed as occurring between the doctor and patient. However, focus is increasingly drawn to a three way interaction [15] that engages doctor, computer and patient in communicative acts. In previous analyses of the consultation the computer's actions have been erased from view, being treated largely as an inanimate object, largely peripheral to the construction of "face" by the doctor or the patient, and peripheral to doctor-patient interaction. Such an approach overlooks the fact that a computer system articulates several very significant influences in the consultation: the ideas the software system designer has of the information flow in the ideal consultation; the ideas that government agencies have of ideal and proscribed information exchanges in the consultation; and the ideas that commercial entities have of what information might be provided for the mutual benefit of the consultation and the commercial entity.

The computer system enables these ideas to be enacted. This performative nature of the face of the computer system provides the basic conditions for the computer's role in the consultation. Indeed, the language of the computer is inherently active. Code that runs on a machine is performative in a much stronger sense than that attributed to language [22].

We therefore begin our analysis with an a priori statement that the scripted behaviour of the computer system that flows from its executable logic. Behaviours follow which manifest in commands and prompts that flow back and forth between the computer system, the doctor and the patient. Also the informational content made legible as a consequence of these scripts, commands and prompts, may all be encapsulated analytically as a Goffmanesque "face" in the consultation.

The aim of this paper is to draw on Goffman's notion of "face" to explore the way in which the actions, visual presentation, and interactions between general practitioners, patients and the computer can imbue the software with its own "face" in the consultation.

\section{Method}

Twenty GPs agreed to allow a single consulting session to be videotaped (2.5-3 h, usually in a morning or an afternoon). We recruited GPs who were significant computer users defined as using clinical software for progress notes, as well as prescribing and test ordering. Effectively, these GPs only generated paper when required by an external organisation. The rationale for doing so was to maximise the degree of interaction available to us, using purposive sampling rather than representative sampling. The use of computers in the consultation is only likely to increase, and this analysis is meant to inform that process. 
Each GP videotaped a single consulting session, which consisted of a variable number of consultations, ranging from 5 to 12. Consent was obtained from each patient before and after the consultation. 192 consultations were witnessed. 34 consultations were lost due to technical difficulties (failure of camera technique or battery life), and 17 due to patient refusal leaving 141 consultations for coding. Non-consenting patients tended to be female, and often requiring an intimate examination. This refusal rate is lower than that previously reported [23].

The detail of the interpretive process has been described elsewhere [24]. Each session of the consultation was digitized into video management software, which allowed the researchers to tag specific micro actions (gaze direction, etc.) in the videos as well as observing the flow of the consultation. This tagging allowed sequential viewing of individual consultations, as well as comparisons across consultations. A general practitioner and a sociologist initially viewed each consultation and then reviewed the consultations in part and as a whole many times as the frameworks developed. First a conceptual framework was developed [25] to apply to subsequent analysis to the videos to observe how the computer affected the interactions.

This analysis represents the findings with regards to the observed actions within the consultation. Within the videos action sequences could be observed that involved interaction with the computer screen, thus the clinical programs used by the GPs. Because we did not have the facility for screen capture, screen activity had to be inferred from what could be seen on the single camera and from the context of the consultation. This was usually not difficult. The sequence of screens for prescribing, or the prompts for immunisations were easily identifiable and reproducible.

Approval for this study was provided by the University of Melbourne Human Research Ethics Committee.

\section{Results}

The mean length of consultations was $12.7 \mathrm{~min}$. Not surprisingly the screen was seen to be the most active agent of the computer, and certainly the one that interacts with both humans in the consultation. Whilst the doctor has access to keyboard and mouse, it was only the screen that exerted significant impact in the interaction. The screen was the primary method by which the computer system as a whole communicated with the humans, was used for a wide variety of functions, and occupied the doctor for significant amounts of time [26]. For the purposes of illustration in the rest of this discussion, the screen shots in this paper are examples only; they are not directly derived from the consultations.

\subsection{Summary screens}

A doctor's entry point to the computer was via a summary screen. Almost universally, this screen was the one on the monitor either at patient entry or when the doctor brought up the record. The only exception to this is discussed in the next section. This screen contained a lot of information with different meanings. The information contained in this early screen was observed to shape the consultation in a myriad of different ways. Absent information (such as demographics or missing smoking status) influenced the beginning of consultations, as did the relative ease of accessing past visits. Doctors were often responding to information on this summary screen.

These screens are all designed to reflect doctor's familiarity with that which they displaced, the common A4 summary sheets prevalent prior to computerisation. The next three figures are typical front/summary screens from three of the programs.

Each of the three programs' summary screens conforms to a basic structure informed by assumed familiarity with the operating system of computers, rather than an assumed familiarity with a paper patient record. There are the cartoonlike icons at the top of the screen, corresponding to common actions such as prescribing and ordering tests. This positioning mirrors the icon placement of most common programs, such as word processors, and facilitates rapid recognition of the common elements, assuming the doctor is familiar with these conventions. Below that are various screens for patient information. The distinction here is that increasingly we are creating an electronic reflection of the patient, the 'patient inscribed', different to the physical patient, the 'patient embodied' [27], Such distinctions are becoming more important the more information doctors accumulate electronically about an individual. It is here that a computer representation of the patient (relevant to their medical history) resides.

Marc Berg [28] argues the case for a mix of free text and forms in clinical packages, based on how humans interact with computer systems. The balance is needed between the ability for the computer to synthesise and interpret information in forms, versus the familiarity with free text entry, and its richness [29]. Each package makes design choices around this issue. That is, each package delegates the tasks required to complete a patient record differently, some giving the computer more responsibility (forms), others giving the doctor more responsibility (free text).

Several differences can also be seen in how the four programs present the inscribed patient information. Again, each program plays an active role, and makes decisions about what information is displayed, and what remains hidden deeper in the program. The program in Fig. 1 for instance, makes very prominent the prescribing information in a window across the screen down at the bottom. However Fig. 2 de-emphasises the patient details when compared to 1 and 3, relegating them to a single line at the top. Fig. 3 ensures the right half of its screen is devoted to past visits, a feature not found on either of the other two. Fig. 1 separates current problems with past history, unlike the other examples. GPs

These are not just simple programming quirks, but represent significant assumptions about what information is important and what is not, and how patient information is best presented. For whilst all the information is contained somewhere in the program, much of it is several clicks away.

\subsection{Prompts}

Second to the interaction with the summary screen, the next most common interaction was with a prompt. Prompts 


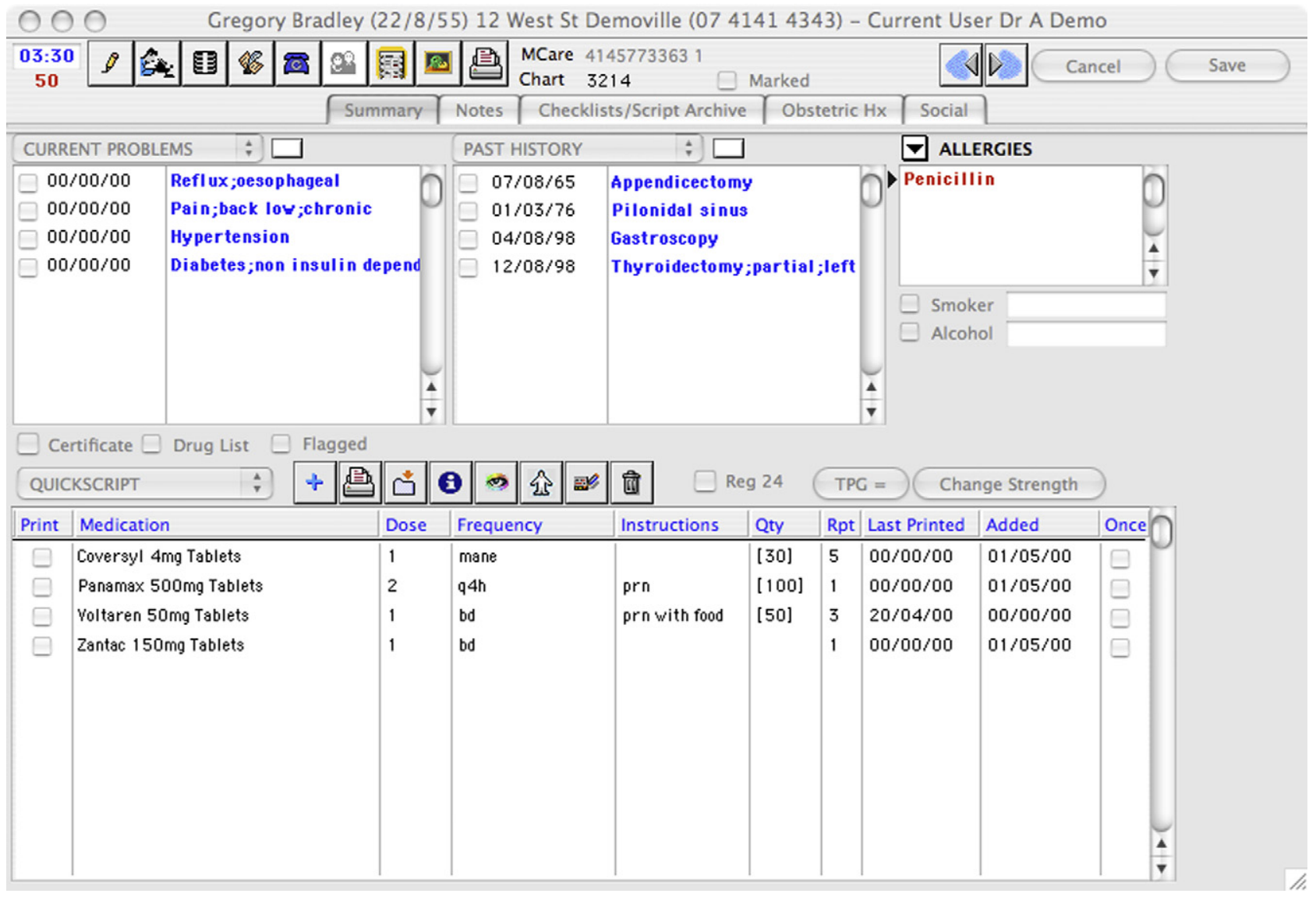

Fig. 1 - Summary screen 1.

occurred at two times in the consultation; at the beginning of the consultation and related to reminders and public health prompts, whilst the next set were generated by data entry during the consultation, such as prescribing (e.g. drug interactions). These prompts actively shaped the beginning in $7 \%$ of the observed consultations. Doctors were often seen to be responding to these prompts in two ways, by either distracting the flow of the patient speech whilst they cleared active prompts, or responding to the prompts (whether active or passive) to distract the flow of the consultation.

Fig. 4 shows us two examples of 'prompt performance', which can be sequential. Once one is cleared, then the second one appears, and so on. These can be described as active prompts that require action and prohibit action in so much as the doctor is unable to proceed until these dialogue boxes are cleared. They are carefully placed so as not to obscure the patient demographic details, but do obscure clinical details, such as past history. Active prompts were clearly observed to be disruptive to the flow of the consultation, and inhibited patient speech whilst drawing gaze of both participants to the computer. In effect the computer became the focus, rather than the patient.

As mentioned earlier, the concept of 'face' is a more encompassing notion that just the physical entity of a face, and includes many social constructs around our projections of ourselves to the world at large. The first interaction with the clinical program represents the first example of how different packages have different "personalities". An important asset of a programme's "personality" is its ability to handle reminders, as well as make suggestions of important health issues. The timing of these reminders is crucial to the flow of the consultation, and to the face the computer presents in the consultation. One program exerts this influence quite actively, through frequent use of dialogue boxes that must be cleared before the consultation can progress. It does this even before the consultation has got underway, with many of these prompts appearing when patient details are first brought up on the screen.

The source of these prompts is worth examining, for they are not generated in isolation by the program. They are, in effect, the manifestation of an external agency influencing the performance of the consultation. The first of the examples is generated by a government policy related to the prescribing restrictions regarding immunisation. The second is part of a government initiative promoting physical activity in the elderly, a preventive health measure. Each is generated by the program in response to the interaction within the program of guidelines linked to the patient's demographic details. Not all of these active prompts are generated by government initiatives though; one program at the time of analysis allowed private company sponsorship of these popups.

In contrast, prompts were dealt with quite differently in a different program, one that passively used colour rather than active dialogue boxes to bring similar information to the 
attention of the doctor. Opening the patient file generated a separate, red window that occupied part of the screen; it does not need to be selected before the program will allow the consultation to continue. Its face therefore intervened in the consultation to enable and provide, rather than requiring or prohibiting.

\subsection{Information recording}

Information recording is a key activity of the computer system, and no consultation occurred without any recording at all. Information recording requires the use of the keyboard, and $5 \%$ of the consultation time was spent exclusively typing information into the system (either history/examination or activities such as prescribing/test ordering). All four programs offer a choice of free text recording of history and examination, or tick-box selection of specific pieces of information (see Fig. 5). Despite expressed concerns about the effects of typing speed, doctors were observed to be much more fluent when typing text that when negotiating tick box selectors, and much more able to maintain patient contact. This particularly applied to the selection of diagnoses for recording. Whilst tickbox selection lacks some of the richness of what may have
Table 1 - Computer behaviours.

\section{Active}

Passive

Informational

Prompting

Distracting

once been written, information recorded in this way (as different from free text) does have the ability to be searched, and used in other areas of the program - thus delegating still more tasks and thus responsibility to the computer system. The height and weights from the examination section may be used in a cardiovascular risk calculator for instance.

\subsection{Effects}

Whilst obvious that prompts would be able to change the flow of the consultation, we were able to categorise several other ways in which the exhibited face was able to do so. The computer could influence the consultation actively or passively, and independently of that categorisation, the computer could exhibit informational behaviour, prompting behaviour or distracting behaviour (Table 1).

The function of face mentioned before - that it is both what is presented to, and what is constructed by the observers,

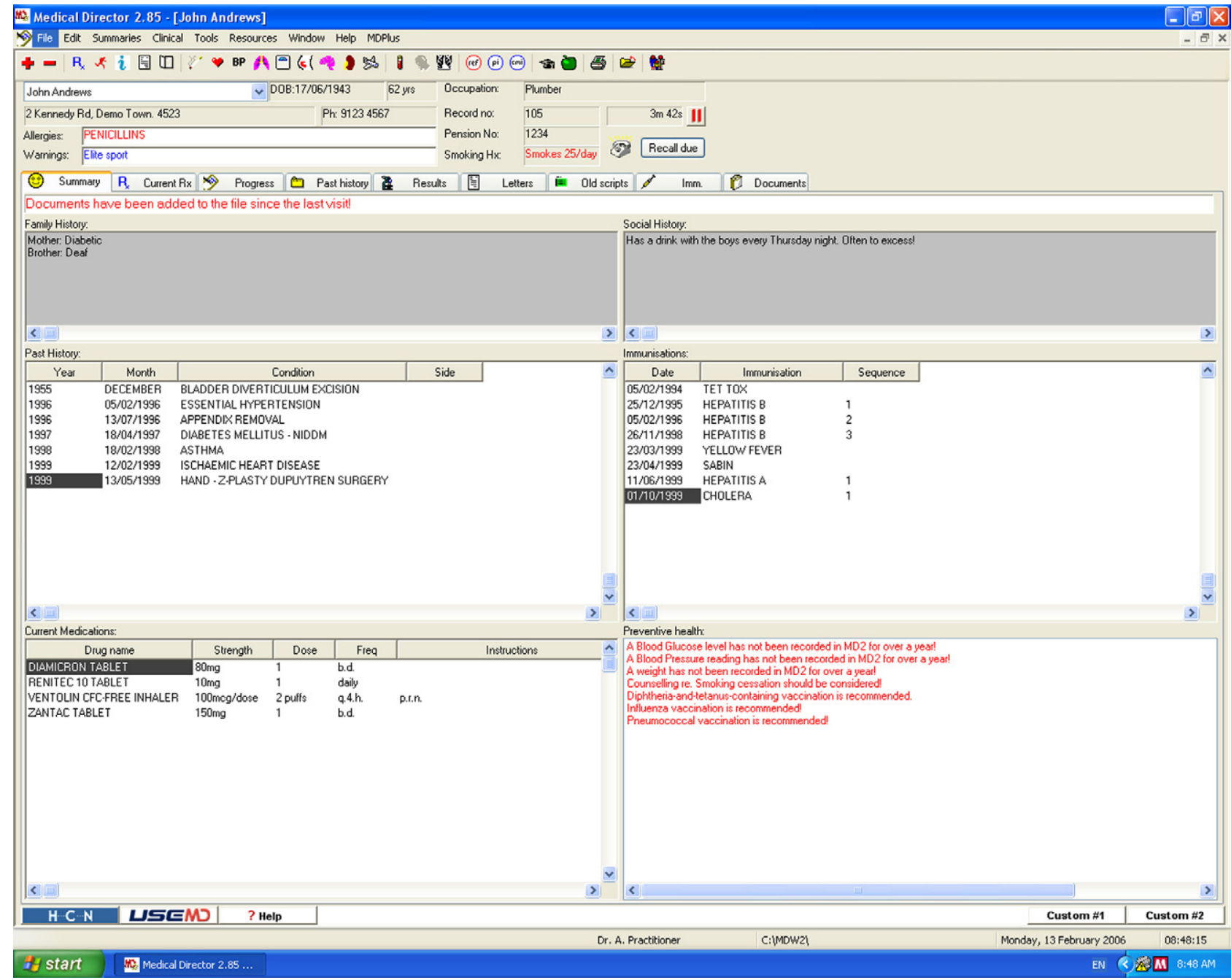

Fig. 2 - Summary screen 2. 


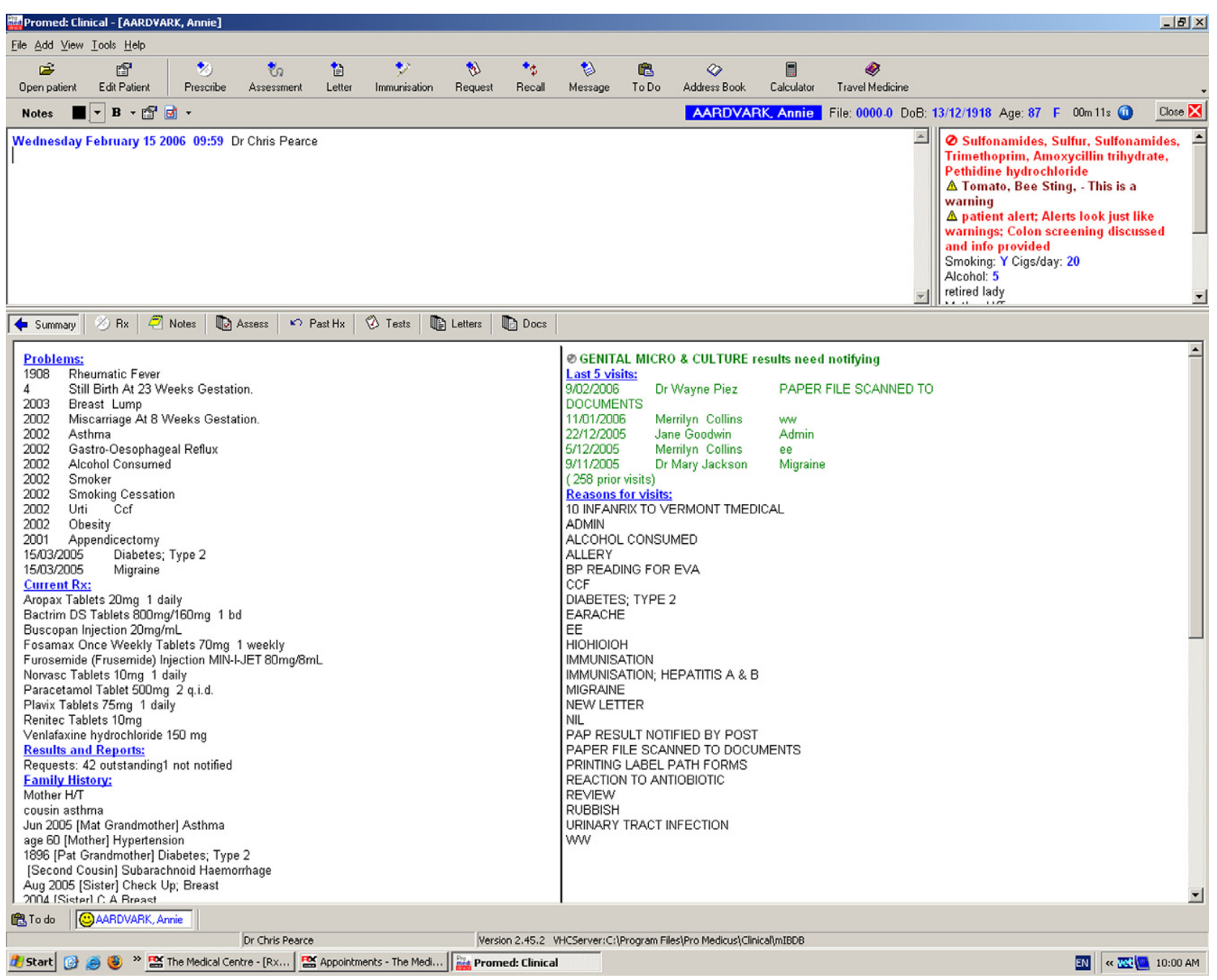

Fig. 3 - Summary screen 3.

allows the same screen to have different consequences according to the context of the consultation. In the following example we can see how this is acted out (Fig. 6).

In the example, the computer system is not offering a prompt, instead it is passively distracting from the flow of the consultation by the doctor noticing that smoking is not recorded in the summary screen, and fixing that deficit - to the detriment of the patients flow of narrative. In other parts of the consultation the computer provided information - 'I can see your last PAP smear was normal' - in addition to the more obvious prompts already discussed.

Where a program provided multiple prompts on patient arrival, the consultation was clearly distracted, whereas programs less reliant on prompts allowed the humans to generate their own agendas. Importantly, this constructed face was the agent that changed the consultation - a creation of both the actions of the computer and the interpretations applied by the humans

\section{Discussion}

A limitation of this study is a lack of detailed screen capture matched with micro-interactions. The lack of this screen capture did not allow detailed analysis of times or incidences of specific behaviours. Such analysis would be important to extend this work, and the principles we describe would inform such research. This study does not attempt to be a comprehensive survey of all software packages available, either here or elsewhere, and is confined to primary care, where the nature of the doctor-patient interaction is different to specialist practices.

The computer can thus be seen to be an active agent in the consultation. There are a variety of drivers for the computer system's performance, important among which are the social forces it brings to the consultation. The computer brings an expression of society into the consultation, in the way in which decisions about preventive health priorities, etc. can be made manifest. No participant in the interaction is value neutral, either human or non-human, and all bring moral, cultural and ethical approaches to their interactions.

There is a structure inherent in the programming of the computer system used in the consultation, and that structure makes a set of value judgments manifest. Young pointed out that health researchers make assumptions, they assume that sick people are rational, when in fact they may not 
a

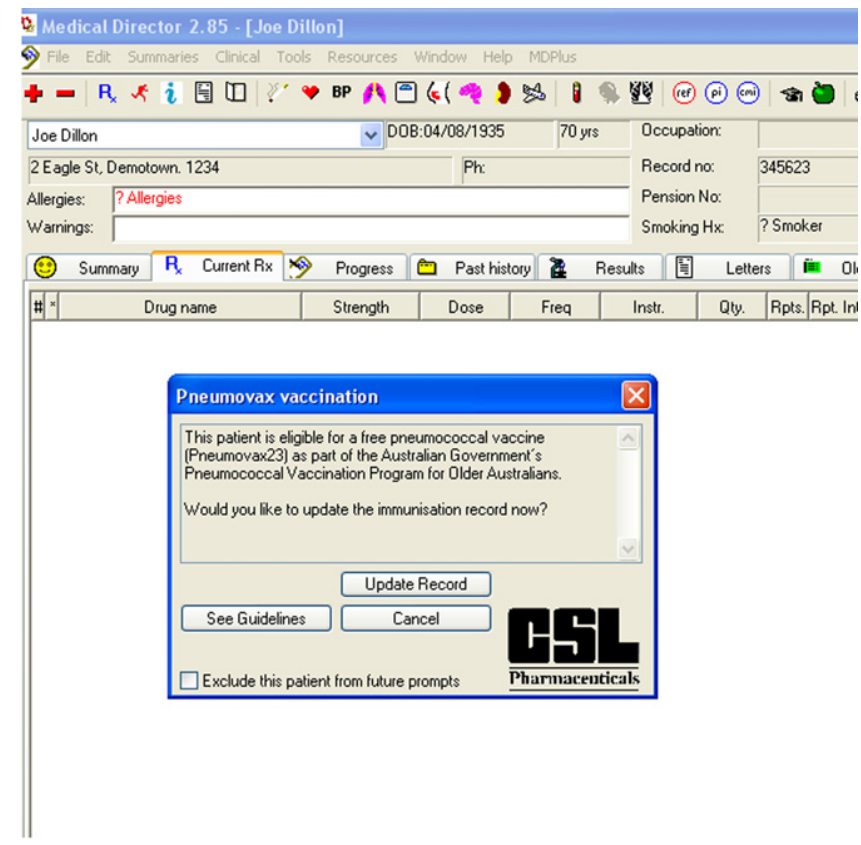

b

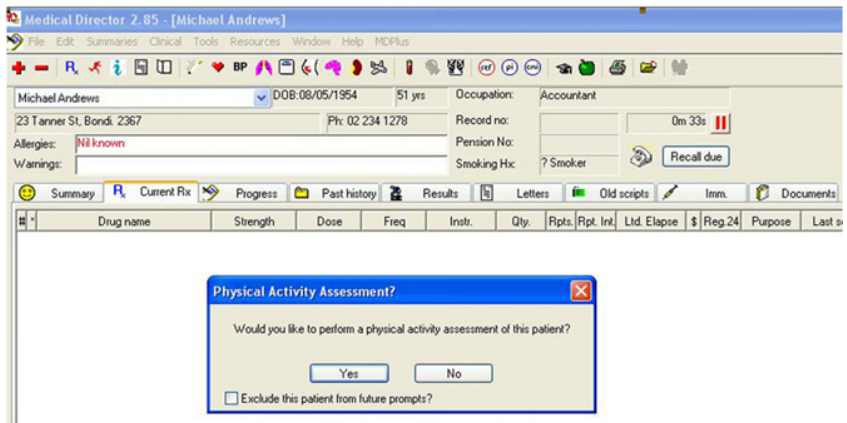

Fig. 4 - Health prompts.

be [30]. In the same vein, programmers make assumptions about the information to be captured, processed and presented through a computer system. There is emphasis on a specific structure for the record, along the lines of history/examination/diagnosis/treatment. This is an imposed structure, and represents a biomedical emphasis on the information. What is presented in the minds of the doctor and patient, is then expressed orally into the consultation, and then must be translated into the medical record [29]. In doing so there is a loss of much valuable information, as the narrative of the consultation is effectively compressed into the structure of the program. Progressively the narrative is compressed until it conforms to a biomedical model. It is important, for instance, to record a diagnosis. All the programs have a facility to prompt for one, creating a coded entry in the record. There is no emphasis on the narrative in the programming, and consequently no specific place to record narrative information. A long story from the patient about how on holidays she developed headaches, which kept her in a hotel room, unable to go to the beach, and missed seeing her grandchild, may simply be recorded as 'migraine', without the accompanying contextual information.
This problem-based structure also assumes a linearity and discreteness to the problems that a patient has, which belies the complexity of general practice. A long consultation with a diabetic patient may be about their housing difficulties, the number of hospital outpatient appointments they have, and coping with a complex drug regimen in the context of blindness and peripheral neuropathy. Such a consultation does not lend itself to fitting the neat little boxes in current programs. The examination section also lends itself to a "tick the box" approach. This is not to argue that these approaches are necessarily a bad thing, simply that by imposing a structure, the programs shape how the interactions in the consultation occur. Through enabling, requiring and prohibiting the form in which information is captured, processed and displayed, and through enabling, requiring and prohibiting the timing of when information is captured, processed and displayed, the computer is capable of imposing a specific structure in the consultation. The current structure that the programs manifest suggests an emphasis on the physical, and on the need to break a patients presenting narrative down into discrete problems, each of which will hopefully have a solution. They represent a social view of the predominance of the 


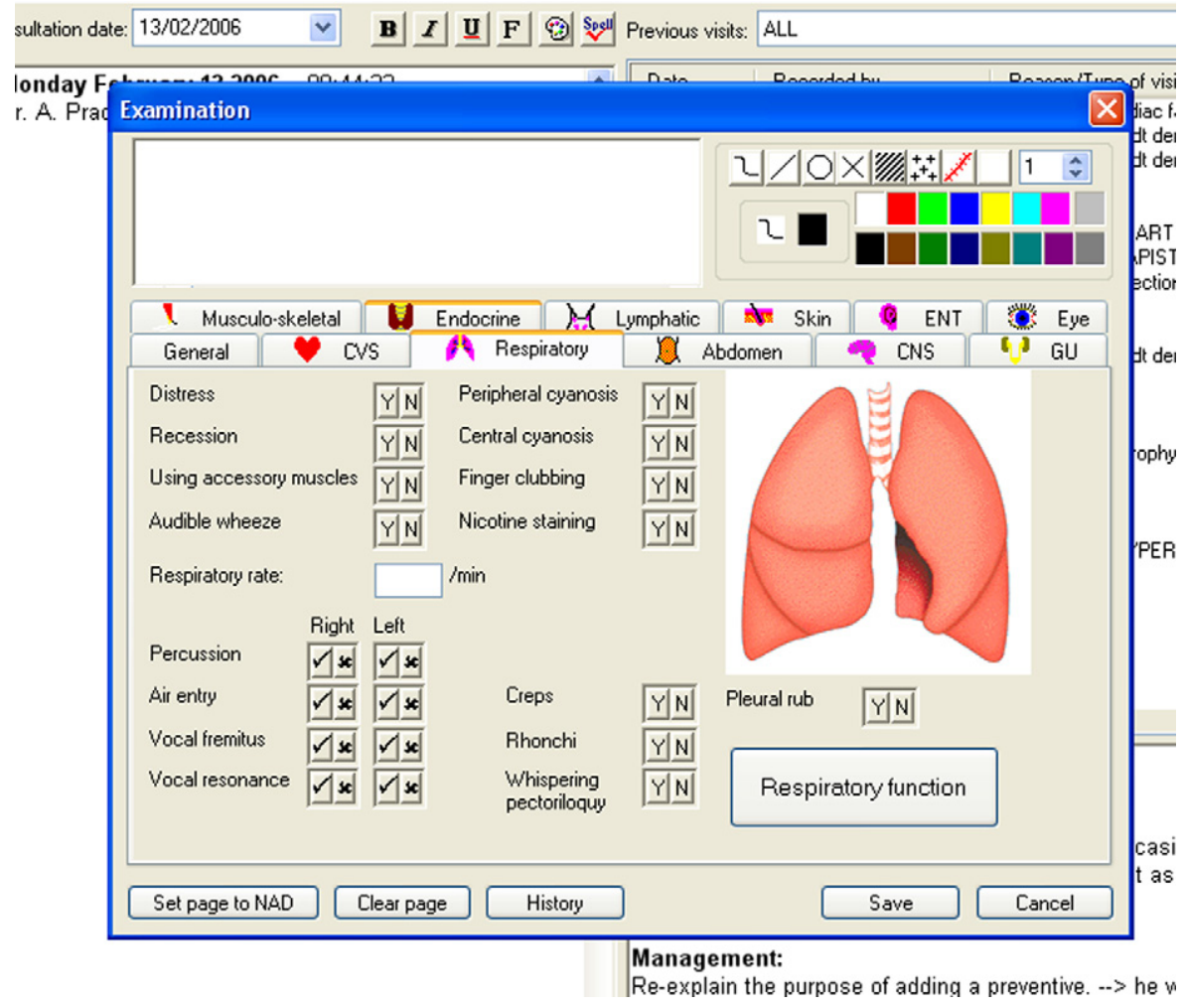

Fig. 5 - Examination screen.

biomedical model. It is complex to record "just wanted a chat" as a diagnosis.

Society, through the agency of government, influences medical interactions. It does so by the imposition of regulations. In the consultation, regulation has a significant impact, and not just on the computer. A not uncommon patient presentation is "I'm just here for my prescriptions" - an attendance determined by the drug regulations, which limit the amount than can be prescribed at any one time. Using the computer for prescribing is the most commonly used part of all clinical packages [1]. Previously, prescriptions were paper based, and unless the doctor recorded the specific information, little in the way of records were kept. "Rx Amoxil" (an antibiotic) was commonly the only written record, with no information on dosing or number of tablets prescribed. Now, the pharmaceutical regulations built into the program can determine the maximum pack sizes and repeats, monitor whether prescriptions are due, and thus use the computer system as a Government proxy for compliance (by querying why the patient has come too early or too late). The regulations monitor the correct diagnoses for authority-only drugs, and facilitate the process of applying for them. With each update, the computer effortlessly changes all the manifestations of those regulations in the computer program, giving an
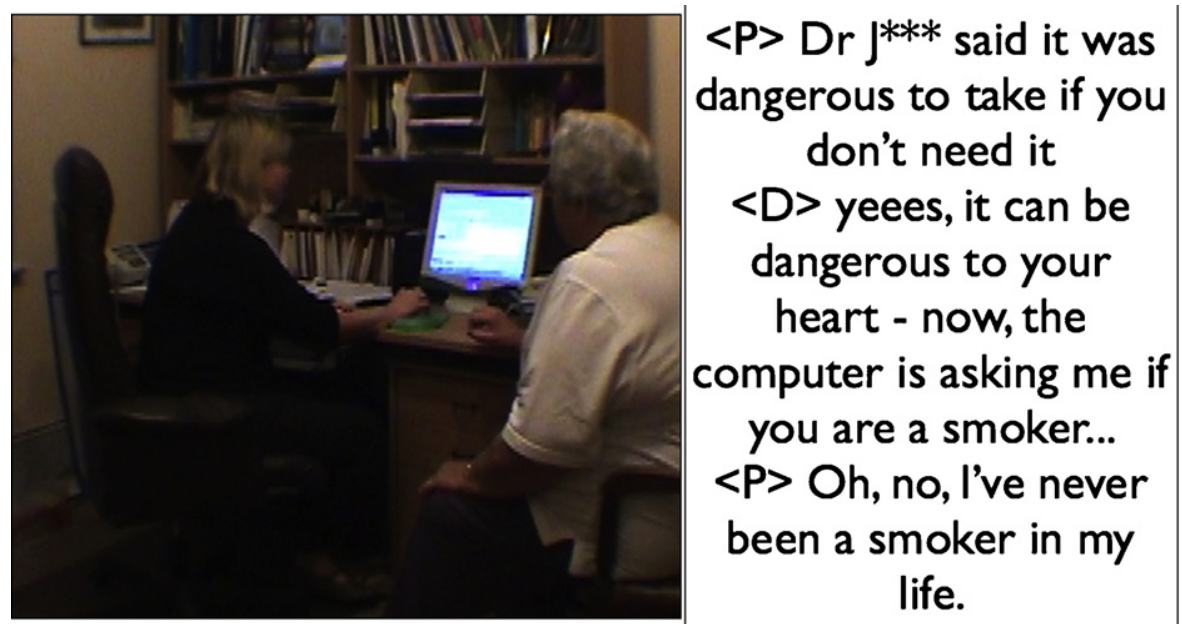

Fig. 6 - Consultation example. 
Summary points

What is already known

- Computers are now prominent in the doctor-patient interaction

- A three way (or triadic) relationship is developing

What this study adds

- Software design makes an impact in the interaction

- This 'face' will shape the consultation into the future

unprecedented means of influencing behaviour. Regulation need not be only governmental, but can also come from professional standards

We know that doctors lessen their use of the computer when the consultation has a significant psychological element [31], we know the computer can influence the beginning of the consultation [32]. We can now show that a computer program itself can express an influence in the consultation, and that that influence is shaped by design decisions expressing themselves according to a complex mix of factors held under the concept of face.

\section{Conclusion}

The computer system has a face that emerges in the consultation. This arises partly in response to the interaction of the patient and doctor, and partly because the computer system chosen brings to the consultation both an inherent performance derived from the fact that it is a computer system and not a paper system, and a performance generated by external forces. This paper is not about what 'ideal' software should look like, but seeks to explain the variety of influences on the software as it informs the consultation, and the variety of influences of the software as it informs the consultation. The more active the mode of the presence of the computer in the consultation, the more patients and doctors will have to adapt their communicative styles in response.

\section{Authors contributions}

CP designed and performed the research. The others provided input into analysis and theory development. CP drew up the first draft, and all other authors contributed and approved the final version.

\section{Conflict of interest statement}

The authors acknowledge that they have no conflicts of interest relevant to this study.

\section{Acknowledgements}

Dr Pearce was supported by an NH\&MRC scholarship, and the research was funded by an RACGP informatics fellowship.

\section{REFERENCES}

[1] D.K. McInnes, D.C. Saltman, M.R. Kidd, General practitioners' use of computers for prescribing and electronic health records: results from a national survey, Med. J Aust. 185 (2) (2006) 88-91.

[2] N. Waring, To what extent are practices 'paperless' and what are the constraints to them becoming more so? Br. J. Gen. Pract. 50 (450) (2000) 46-47.

[3] C. Watkins, I. Harvey, C. Langley, A. Faulkner, S. Gray, General practitioners' use of computers during the consultation, Br. J. Gen. Pract. 49 (442) (1999) 381-383.

[4] A.K. Jha, D. Doolan, D. Grandt, T. Scott, D.W. Bates, The use of health information technology in seven nations, Int. J. Med. Inform. 77 (12) (2008) 848-854.

[5] A.K. Jha, T.G. Ferris, K. Donelan, C. DesRoches, A. Shields, S. Rosenbaum, D. Blumenthal, How common are electronic health records in the United States? A summary of the evidence, Health Aff. (Millwood) 25 (6) (2006) w496-w507.

[6] J.C. Martin, R.F. Avant, M.A. Bowman, J.R. Bucholtz, J.R. Dickinson, K.L. Evans, L.A. Green, D.E. Henley, W.A. Jones, S.C. Matheny, et al., The future of family medicine: a collaborative project of the family medicine community, Ann. Fam. Med. 2 (Suppl 1) (2004) S3-S32.

[7] J.C. Wyatt, F. Sullivan, eHealth and the future: promise or peril? BMJ 331 (7529) (2005) 1391-1393.

[8] M. Kidd, The computerisation of australian general practice 1998-2001 - what did we get for AU\$15,000,000, Inform. Prim. Care 10 (1) (2002) 25-29.

[9] L. Stoicu-Tivadar, V. Stoicu-Tivadar, Human-computer interaction reflected in the design of user interfaces for general practitioners, Int. J. Med. Inform. 75 (3-4) (2006) 335-342.

[10] P. Kumarapeli, S. de Lusignan, P. Koczan, B. Jones, I. Sheeler, The feasibility of using UML to compare the impact of different brands of computer system on the clinical consultation, Inform. Prim. Care 15 (4) (2007) 245-253.

[11] C. Pearce, H. Walker, C. O'Shea, A visual study of computers on doctors' desks, Inform. Prim. Care 16 (2) (2008) 111-117.

[12] K. Häyrinen, K. Saranto, P. Nykänen, Definition, structure, content, use and impacts of electronic health records: a review of the research literature, Int. J. Med. Inform. 77 (5) (2008) 291-304.

[13] A. Hoerbst, C.D. Kohl, P. Knaup, E. Ammenwerth, Attitudes and behaviors related to the introduction of electronic health records among Austrian and German citizens, Int. J. Med. Inform. 79 (2) (2010) 81-89.

[14] A. Hoerbst, E. Ammenwerth, Electronic health records. A systematic review on quality requirements, Methods Inf. Med. 49 (4) (2010) 320-336.

[15] D. Scott, I. Purves, Triadic relationship between doctor, computer and patient, Interacting Comput. 8 (4) (1996) 347-363.

[16] W. Ventres, S. Kooienga, R. Marlin, N. Vuckovic, V. Stewart, Clinician style and examination room computers: a video ethnography, Fam. Med. 37 (4) (2005) 276-281.

[17] E. Goffman, Interaction Ritual: Essays on Face-to-face Behavior, 1st Pantheon Books edn., Pantheon Books, New York, 1982. 
[18] A. Als, The desk-top computer as a magic box: patterns of behaviour connected with the desk-top computer; GPs' and patients' perceptions, Fam. Pract. 14 (1) (1997) 17-23.

[19] C. Pearce, M. Arnold, C. Phillips, S. Trumble, K. Dwan, The patient and the computer in the primary care consultation, J. Am. Med. Inform. Assoc. 18 (2) (2011) 138-142.

[20] I.N. Purves, Facing future challenges in general practice: a clinical method with computer support, Fam. Pract. 13 (6) (1996) 536-543.

[21] E.G. Mishler, The Discourse of Medicine: Dialectics of Medical Interviews, Ablex Pub. Corp, Norwood, NJ, 1984

[22] N.K. Hayles, My Mother was a Computer: Digital Subjects and Literary Texts, University of Chicago Press, Chicago, 2005.

[23] T. Coleman, Using video-recorded consultations for research in primary care: advantages and limitations, Fam. Pract. 17 (5) (2000) 422-427.

[24] C. Pearce, K. Dwan, C. Phillips, M. Arnold, Analysing the doctor-patient-computer relationship: the use of video data, Inform. Prim. Care 14 (4) (2007) 221-226.

[25] C. Pearce, K. Dwan, M. Arnold, C. Phillips, S. Trumble, Doctor, patient and computer-a framework for the new consultation, Int. J. Med. Inform. 78 (1) (2009) 32-38.
[26] D. Bui, C. Pearce, E. Deveny, T. Liaw, Computer use in general practice consultations, Aust. Fam. Physician 34 (5) (2005) 400.

[27] J.D. Robinson, Getting down to business - talk, gaze, and body orientation during openings of doctor-patient consultations, Hum. Commun. Res. 25 (1) (1998) 97-123.

[28] M. Berg, C. Langenberg, I. vander Berg, J. Kwakkernaat, Considerations for sociotechnical design: experiences with an electronic patient record in a clinical context, Int. J. Med. Inform. 52 (1-3) (1998) 243-251.

[29] S. Kay, I. Purves, The electronic medical record and "the story stuff": a narrativisitic model, in: T.H.B. Greenhalgh (Ed.), Narrative Based Medicine, 1st ed., BMJ Books, London, 1998, pp. 185-201.

[30] A. Young, When rational men fall sick: an inquiry into some assumptions made by medical anthropologists, Cult. Med. Psychiatry 5 (1981) 317-335.

[31] W.S. Chan, M. Stevenson, K. McGlade, Do general practitioners change how they use the computer during consultations with a significant psychological component? Int. J. Med. Inform. 77 (8) (2008) 534-538.

[32] C. Pearce, S. Trumble, M. Arnold, K. Dwan, C. Phillips, Computers in the new consultation: within the first minute, Fam. Pract. 25 (3) (2008) 202-208. 